\title{
Bożena Piątek
}

\section{ON THE SINCOV FUNCTIONAL EQUATION}

\begin{abstract}
The Riemann integral, the Hukuhara differences and derivatives will be applied to obtain solutions of the Sincov functional equation.
\end{abstract}

\section{Definition of Riemann integral and its properties}

Let $(C,+)$ be an Abelian semigroup with the identity element and let the operation : : $[0, \infty) \times C \rightarrow C$ of multiplication of elements of $C$ by nonnegative numbers $[0, \infty) \times C \ni(\lambda, s) \rightarrow \lambda s \in C$ be given. A triple $(C,+, \cdot)$ is said to be an abstract convex cone if the operations + and . satisfy the following properties

$$
\lambda(s+t)=\lambda s+\lambda t, \quad(\lambda+\mu) s=\lambda s+\mu s, \quad \lambda(\mu s)=(\lambda \mu) s, \quad 1 \cdot s=s
$$

for every $s, t \in C$ and $\lambda \geq 0, \mu \geq 0$.

If $s, t, t^{\prime} \in C, s+t=s+t^{\prime}$ implies that $t=t^{\prime}$, then $C$ is said to satisfy the cancellation law. Suppose further that a metric $\rho$ is given in $C$ and $(C, \rho)$ is a complete metric space. Moreover, let $\rho$ be translation invariant, i.e.,

$$
\rho\left(s+t, s+t^{\prime}\right)=\rho\left(t, t^{\prime}\right) \text { for all } s, t, t^{\prime} \in C
$$

and positively homogeneous, i.e.,

$$
\rho(\lambda s, \lambda t)=\lambda \rho(s, t) \text { for all } s, t \in C \text { and } \lambda \geq 0 .
$$

A metric abstract convex cone with all above properties is said to be a complete metric cone.

Let $f:[a, b] \rightarrow C$ be a function defined on interval $[a, b] \subset \mathbb{R}$ with values in a complete metric cone $C$. A set $\Delta=\left\{x_{0}, x_{1}, \ldots, x_{n}\right\}$, where

$$
a=x_{0}<x_{1}<\ldots<x_{n}=b,
$$

is said to be a partition of $[a, b]$. For given partition $\Delta$ we set $\delta(\Delta)=$ $\max \left\{x_{i}-x_{i-1}: i \in\{1, \ldots n\}\right\}$. For a partition $\Delta$ and for a system $\tau=$

1991 Mathematics Subject Classification: 39B52, 26A42, 28B20.

Key words and phrases: Sincov functional equation, Riemann integral, abstract convex cone, multifunctions. 
$\left(\tau_{1}, \ldots, \tau_{n}\right)$ of intermediate points $\tau_{i} \in\left[x_{i-1}, x_{i}\right]$ we form the Riemann sum

$$
S(\Delta, \tau)=\sum_{i=1}^{n}\left(x_{i}-x_{i-1}\right) f\left(\tau_{i}\right)
$$

Let $I$ be an element of $C$. If for each $\varepsilon>0$ there exists $\delta>0$ such that

$$
\rho(I, S(\Delta, \tau))<\varepsilon
$$

for every partition $\Delta=\left\{x_{0}, x_{1}, \ldots, x_{n}\right\}$ such that $\delta(\Delta)<\delta$ and for all intermediate points $\tau_{i} \in\left[x_{i-1}, x_{i}\right], i \in\{1, \ldots, n\}$, then $I$ is said to be the Riemann integral of $f$ over $[a, b]$ and it is denoted by $\int_{a}^{b} f(x) d x:=I$. In this case $f$ is said to be Riemann integrable.

An ordinary difference is not defined in a cone. Thus proofs of some properties of the Riemann integral are not always the same as for real functions. Nevertheless it is able to prove that bounded function $f:[a, b] \rightarrow C$ which is continuous expect a finite number of points of $[a, b]$ is Riemann integrable. Also we can prove that the integrability of $f$ on $[a, b]$ implies the integrability of this function on each subinterval $[c, d] \subset[a, b]$ and the equality

$$
\int_{a}^{b} f(x) d x=\int_{a}^{c} f(x) d x+\int_{c}^{b} f(x) d x
$$

holds for all $c \in(a, b)$.

\section{Definition of derivative}

In an Abelian semigroup we are able to define a difference of two elements of $C$. For given $s, t \in C$, an element $u \in C$ is said to be the difference $t-s$ if $t=s+u$ (see [5, p. 210]). If an abstract convex cone satisfies the cancellation law and the difference exists, then it is unique. The definition of the derivative given below is due to M. Hukuhara (cf. [5, pp. 210-211]). Let $f:[a, b] \rightarrow C$ and let $x_{0} \in(a, b) . f$ is differentiable at $x_{0}$ if there exist the limits

$$
\lim _{x \rightarrow x_{0}^{+}} \frac{f(x)-f\left(x_{0}\right)}{x-x_{0}}=: f_{+}^{\prime}\left(x_{0}\right)
$$

and

$$
\lim _{x \rightarrow x_{0}^{-}} \frac{f\left(x_{0}\right)-f(x)}{x_{0}-x}=: f_{-}^{\prime}\left(x_{0}\right)
$$

and they are equal. Then the derivative of $f$ at $x_{0}$ is defined by $f^{\prime}\left(x_{0}\right):=$ $f_{+}^{\prime}\left(x_{0}\right)=f_{-}^{\prime}\left(x_{0}\right)$. In this definition it is assumed that the differences $f(x)-$ $f\left(x_{0}\right)$ and $f\left(x_{0}\right)-f(x)$ exist for all $x>x_{0}$ and $x<x_{0}$, respectively, sufficiently close to $x_{0}$. We say that $f$ is differentiable at $a$ if there exists $f_{+}^{\prime}(a)$ and $f$ is differentiable at $b$ if $f_{-}^{\prime}(b)$ exists. In these cases we set $f^{\prime}(a):=f_{+}^{\prime}(a)$ and $f^{\prime}(b):=f_{-}^{\prime}(b)$. 
It can be shown that if $f:[a, b] \rightarrow C$ is continuous and $C$ is a complete metric cone, then $g:[a, b] \rightarrow C$ defined by the formula

$$
g(x)=\int_{a}^{x} f(y) d y
$$

is differentiable on $[a, b]$ and $g^{\prime}=f$.

We are also able to prove that if $f, g:[a, b] \rightarrow C$ are continuous and $g_{+}^{\prime}(x)=f(x), x \in[a, b)$, then the difference $g(b)-g(a)$ exists and the formula $\int_{a}^{b} f(x) d x=g(b)-g(a)$ holds. Moreover, $g$ is differentiable on $[a, b]$.

\section{Sincov functional equation}

The functional equation

$$
f(x, y)+f(y, z)=f(x, z)
$$

was studied by D. M. Sincov in 1903 . Assume that $M$ is a nonempty set and let $(X,+)$ be an Abelian group. It is known (see e.g. $[1$, p. 223]) that the general solution of $(1)$ is of the form

$$
f(x, y)=g(y)-g(x)
$$

where $g: M \rightarrow X$ is an arbitrary function.

Now let $(C,+)$ be a semigroup satisfying the cancellation law. Assume that $D$ is a nonempty subset of $M \times M$ for which the following condition holds

$$
(x, y) \in D \wedge(y, z) \in D \Rightarrow(x, z) \in D .
$$

Suppose that $f: D \rightarrow C$ is a solution of (1). Let us take $(x, y) \in D$, $(a, x) \in D$. Then $(a, y) \in D$ and there exists the difference $f(a, y)-f(a, x)$. Thus $f$ is of the form (2) for all $(x, y) \in D$, where $g(x):=f(a, x)$.

Conversely, if $g: M \rightarrow C$ is an arbitrary function such that there exist all differences $g(y)-g(x)$ for every $(x, y) \in D$, then $f(x, y):=g(y)-g(x)$ is a solution of (1) in $D$. Thus we have the following theorem.

THEOREM 1. Let $(C,+)$ be a semigroup satisfying the cancellation law. Assume that $M$ is a set and $D$ is a nonempty subset of $M \times M$ such that condition (3) holds. Then a function $f: D \rightarrow C$ is a solution of (1) if and only if there exists a function $g: M \rightarrow C$ such that all differences $g(y)-g(x)$ exist and $f(x, y)=g(y)-g(x)$ for $(x, y) \in D$.

In the sequel we denote by $T$ the set

$$
\left\{(x, y) \in \mathbb{R}^{2}: 0 \leq x \leq y<\infty\right\} .
$$

THEOREM 2. Assume that $C$ is a complete metric cone and $g:[0, \infty) \rightarrow C$ is a Riemann integrable function on every interval $[a, b] \subset[0, \infty)$. Then 
the function

$$
f(x, y):=\int_{x}^{y} g(t) d t
$$

defined on $T$ is a solution of (1).

Proof. Let $0 \leq x \leq y \leq z<\infty$. We have by the additivity of an integral

$$
f(x, y)+f(y, z)=\int_{x}^{y} g(t) d t+\int_{y}^{z} g(t) d t=\int_{x}^{z} g(t) d t=f(x, z)
$$

THEOREM 3. Let $C$ be a complete metric cone. If $f: T \rightarrow C$ is a solution of (1) and if $\frac{\partial}{\partial y} f(0, \cdot)$ exists and is continuous then

$$
f(x, y)=\int_{x}^{y} \frac{\partial}{\partial y} f(0, t) d t
$$

holds true for all $(x, y) \in T$.

Proof. Define $h: T \rightarrow C$ by

$$
h(x, y):=\int_{x}^{y} \frac{\partial}{\partial y} f(0, t) d t, \quad(x, y) \in T
$$

Since $f(0, \cdot)$ is an antiderivative of $\frac{\partial}{\partial y} f(0, \cdot)$ and $f$ is a solution of (1), we obtain

$$
h(x, y)=f(0, y)-f(0, x)=f(x, y)
$$

for all $(x, y) \in T$.

\section{Riemann integral of multifunction}

Let $X$ be a real Banach space. Denote by $\operatorname{clb}(X)$ the set of all nonempty convex closed bounded subsets of $X$. For given $A, B \in \operatorname{clb}(X)$, we set $A+B=\{a+b: a \in A, b \in B\}, \lambda A=\{\lambda a: a \in A\}$ for $\lambda \geq 0$ and $A \stackrel{*}{+} B=$ $c l(A+B)=\operatorname{cl}(\operatorname{cl} A+\operatorname{cl} B)$, where $c l A$ means the closure of $A$ in $X$. It is easy to see that $(c l b(X), \stackrel{*}{+}, \cdot)$ is an abstract convex cone with the identity element $\{0\}$. If $A, B, C \in \operatorname{clb}(X)$, then the equality $A \stackrel{*}{+} C=B \stackrel{*}{+} C$ implies $A=B$, thus $c l b(X)$ with addition ${ }^{*}$ satisfies the cancellation law (see [6] and [10]).

The abstract convex cone $\operatorname{clb}(X)$ is a metric space with the Hausdorff metric $d$ defined by the relation

$$
d(A, B)=\max \{e(A, B), e(B, A)\},
$$

where $e(A, B)=\sup _{a \in A}\left(\inf _{b \in B}\|a-b\|\right)$. The metric space $(c l b(X), d)$ is complete (see e.g. [2, Theorem II-3, p. 40]). Moreover, the Hausdorff metric 
$d$ is translation invariant since

$$
d(A \stackrel{*}{+} C, B \stackrel{*}{+} C)=d(A+C, B+C)=d(A, B)
$$

(cf. [3, Lemma 2.2] and [6, Lemma 3]) and positively homogeneous

$$
d(\lambda A, \lambda B)=\lambda d(A, B)
$$

for all $\lambda \geq 0$ and $A, B, C \in \operatorname{clb}(X)$ (cf. [3, Lemma 2.2]). Consequently $\operatorname{clb}(X)$ is a complete metric cone.

Let $F$ be a multifunction on an interval $[a, b]$ with convex closed bounded values in $X$. We can define the Riemann integral of the multifunction on $[a, b]$ like in part 1 . In this case the Riemann sum $S(\Delta, \tau)$ has the form

$$
S(\Delta, \tau)=\left(x_{1}-x_{0}\right) F\left(\tau_{1}\right) \stackrel{*}{+} \ldots \stackrel{*}{+}\left(x_{n}-x_{n-1}\right) F\left(\tau_{n}\right) .
$$

EXAMPLE. Let $\left[c_{1}, d_{1}\right],\left[c_{2}, d_{2}\right]$ be arbitrary intervals on the line. It is known that

$$
d\left(\left[c_{1}, d_{1}\right],\left[c_{2}, d_{2}\right]\right)=\max \left\{\left|c_{1}-c_{2}\right|,\left|d_{1}-d_{2}\right|\right\} .
$$

Let $f, g:[a, b] \rightarrow \mathbb{R}$ be real functions such that $f \leq g$. It is not difficult to verify that multifunction $F:[a, b] \rightarrow c l b(\mathbb{R}), F(x)=[f(x), g(x)], x \in[a, b]$ is Riemann integrable on $[a, b]$ if and only if the both functions $f$ and $g$ are Riemann integrable on this interval and the equality

$$
\int_{a}^{b} F(x) d x=\left[\int_{a}^{b} f(x) d x, \int_{a}^{b} g(x) d x\right]
$$

holds.

The Riemann integral for multifunctions with nonempty compact convex values in $\mathbb{R}^{n}$ was introduced by Alexander Dinghas [4] in 1956 and by Masuo Hukuhara [5] in 1967. Its some applications can be found in papers of Dmitry B. Silin (see e.g. [7], [8]). The paper [9] of D. B. Silin contains some version of the Riemann integral. For continuous multifunctions with values as above the integral coincides with the Riemann one defined by Dinghas and Hukuhara (ef. [9, p. 133]). Further papers that deal with the problems of set-valued differentiation and integration are quoted in [9].

Let $A, B, C \in \operatorname{clb}(X)$. A set $C$ is said to be the Hukuhara difference of $A$ and $B$, i.e., $C=A^{*}-B$ if $A=B \stackrel{*}{+} C$ (see [5, p. 210] for convex compact sets $A, B, C$ ). By Rådström's theorem (see [6] and [10]) it follows that if the difference $A^{*}-B$ exists, then it is exactly one.

The differentiability of multifunction $F:[a, b] \rightarrow c l b(X)$ is understood as in part 2. 


\section{Multivalued version of Sincov functional equation}

Let $X$ be a Banach space. Consider equation (1)

$$
F(x, y) \stackrel{*}{+} F(y, z)=F(x, z)
$$

for $0 \leq x \leq y \leq z<\infty$, where $F: T \rightarrow c l b(X)$ and

$$
T:=\left\{(x, y) \in \mathbb{R}^{2}: 0 \leq x \leq y<\infty\right\} .
$$

Theorems 1-3 can be reformulated in the following way.

Theorem $1^{\prime}$. Assume that $X$ is a Banach space. If $P:[0, \infty) \rightarrow \operatorname{clb}(X)$ is a multifunction such that the Hukuhara difference $F(x, y):=P(y) \stackrel{*}{-} P(x)$ exists for all $(x, y) \in T$, then the multifunction $F: T \rightarrow c l b(X)$ satisfies (4) in $T$. Conversely, if $F: T \rightarrow \operatorname{clb}(X)$ is a solution of (4) in $T$, then there exists the Hukuhara difference $F(x, z){ }^{*} F(x, y)$ for every $0 \leq x \leq y \leq z<\infty$ and

$$
F(y, z)=F(x, z) \stackrel{*}{-} F(x, y)
$$

for all $0 \leq x \leq y \leq z<\infty$.

REMARK 1. If $F: T \rightarrow \operatorname{clb}(X)$ is a solution of (4), then

$$
F(x, z)^{*}-F(x, y)=F(0, z) \stackrel{*}{-} F(0, y)
$$

for all $0 \leq x \leq y \leq z<\infty$.

TheOrem $2^{\prime}$. Assume that $X$ is a Banach space and $G:[0, \infty) \rightarrow \operatorname{clb}(X)$ is a Riemann integrable multifunction on every interval $[a, b] \subset[0, \infty)$. Then the multifunction

$$
F(x, y):=\int_{x}^{y} G(t) d t
$$

defined on $T$ is a solution of (4).

Theorem $3^{\prime}$. Assume that $X$ is a Banach space. If $G:[0, \infty) \rightarrow \operatorname{clb}(X)$ is continuous then the multifunction

$$
F(x, y):=\int_{x}^{y} G(t) d t
$$

satisfies (4) and the derivative $\frac{\partial F}{\partial y}(0, y)$ with respect to the second variable of $F(0, \cdot)$ exists and $\frac{\partial F}{\partial y}(0, y)=G(y), y \geq 0$. Conversely, if $F: T \rightarrow c l b(X)$ is a solution of (4) and if the derivative $\frac{\partial F}{\partial y}(0, \cdot)$ exists and it is continuous 
on $[0, \infty)$, then the formula.

$$
F(x, y)=\int_{x}^{y} \frac{\partial F}{\partial y}(0, t) d t
$$

holds true for all $(x, y) \in T$.

\section{References}

[1] J. Aczél, Lecture on Functional Equations and Their Applications, Academic Press, New York and London, 1966.

[2] C. Castaing, M. Valadier, Convex Analysis and Mesurables Multifunctions, Lecture Notes in Math. 580, Springer-Verlag, Berlin, Heidelberg, New York, 1977.

[3] F. S. de Blasi, On the differentiability of multifunctions, Pacific J. Math. 66 (1976), 67-81.

[4] A. Dinghas, Zum Minkowskischen Integralbegriff abgeschlossener Mengen, Math. Z. 66 (1956), 173-188.

[5] M. Hukuhara, Intégration des applications mesurables dont la valeur est un compact convexe, Funkcial. Ekvac. 10 (1967), 205-223.

[6] H. Rådström, An embedding theorem for spaces of convex sets, Proc. Amer. Math. Soc. 3 (1952), 165-169.

[7] D. B. Silin, On variation and Riemann integrability of optimal control in linear systems, Dokl. Akad. Nauk SSSR 257 (1981), 548-550 (in Russian).

[8] D. B. Silin, Riemann integrability of the optimal control in linear time-optimality problems, Izv. Akad. Nauk SSSR Ser. Mat. 48 (1984), no. 4, 854-864 (in Russian).

[9] D. B. Silin, On set-valued differentiation and integration, Set-Valued Analysis 5 (1997), 107-146.

[10] R. Urbański, A generalization of Minkowski-Rådström-Hörmander theorem, Bull. Akad. Pol. Sci. 24(9) (1976), 709-715.

INSTITUTE OF MATHEMATICS

SILESIAN UNIVERSITY OF TECHNOLOGY

Kaszubska 23

44-100 GLIWICE, POLAND

E-mail:Bozena.Piatek@polsl.pl

Received May 17, 2004; revised version May 30, 2005. 
